Review

\title{
Prognostic value of tricuspid regurgitation
}

\author{
Pier Pasquale Leone ${ }^{1,2, *}$, Mauro Chiarito ${ }^{1,2}$, Damiano Regazzoli ${ }^{2}$, Marta Pellegrino ${ }^{2}$, \\ Lorenzo Monti $^{2}$, Beniamino Pagliaro ${ }^{2}$, Ferdinando Loiacono ${ }^{2}$, Giulio Stefanini ${ }^{1,2}$, \\ Daniela Pini ${ }^{2}$, Bernhard Reimers ${ }^{2}$, Antonio Colombo ${ }^{1,2}$, Azeem Latib ${ }^{3}$, Antonio Mangieri ${ }^{2}$ * \\ ${ }^{1}$ Department of Biomedical Sciences, Humanitas University, 20090 Pieve Emanuele-Milan, Italy \\ ${ }^{2}$ Cardio Center, IRCCS Humanitas Research Hospital, 20089 Rozzano-Milan, Italy \\ ${ }^{3}$ Division of Cardiology, Montefiore Medical Center, Bronx, NY 10467-2401, USA \\ *Correspondence: pierpasquale.leone@gmail.com (Pier Pasquale Leone); antonio.mangieri@gmail.com (Antonio Mangieri) \\ Academic Editor: Alexander Lauten \\ Submitted: 26 December 2021 Revised: 24 January 2022 Accepted: 27 January 2022 Published: 22 February 2022
}

\begin{abstract}
Tricuspid regurgitation (TR) has a considerable prevalence in the overall population, that further increases in selected categories of patients. Three morphologic types of TR prevail, namely primary, secondary and atrial TR, mostly, but not always, occurring in different subsets of patients. Recent evidences demonstrate a negative impact of TR on outcomes, irrespective of etiology and even when less than severe in grading. Unfortunately, current surgical standards are void of strong prospective evidence of positive impact on clinical outcomes. While on one hand recent advances in diagnosis and risk stratification of patients with TR are shedding light onto the population that may benefit from intervention and its appropriate timing, on the other hand the arrival on stage of percutaneous treatment options is widening even more the therapeutic options for such population. In this review we will address and discuss the available evidence on the prognostic impact of TR in different clinical contexts encountered in practice.
\end{abstract}

Keywords: tricuspid regurgitation; valvular heart disease; right ventricle; heart failure

Progressive accrual of insights into the prevalence, pathophysiology, natural history and clinical relevance of tricuspid regurgitation (TR) has been witnessed in recent years [1-4], and treatment of TR gradually shifted from a conservative to a more interventional therapeutic and preventive approach. Advances in echocardiographic techniques have been accomplices for such radical changes in management. Three different types of TR can be distinguished, that is primary, secondary and atrial TR (Fig. 1). While primary TR is due to an abnormality of the tricuspid valve (TV) apparatus, TR may be caused by dilation of TV annulus, right ventricular (RV) remodeling and leaflet tethering secondary to left heart disease and pulmonary hypertension (secondary TR), or caused by atrial fibrillation (AF) and right atrium (RA) remodeling (atrial TR) [4-6]. Secondary TR is the most common manifestation of TV disease, and has traditionally been considered a benign disease manageable with diuretic therapy and by addressing left heart disease. On the other hand, growing evidence has shown deleterious outcomes of untreated TR irrespective of left or right ventricular function and pulmonary hypertension in the surgical, percutaneous, and heart failure populations studied [7,8]. In light of this evidence and the mortality risk associated with surgical intervention in candidate patients at high risk, novel transcatheter devices have been developed to address this unmet clinical need. This review will address the available evidence concerning the prognostic impact of TR in the different contexts with a particular focus on secondary TR (Table 1, Ref. [2,8-23]).

\section{Natural history of tricuspid regurgitation}

TR is a common echocardiographic finding, reported in up to 70 to $90 \%$ of adults [1] and clinically relevant TR is found in approximately $4 \%$ of subjects aged 75 years [2]. Historically, the management of TR by the medical community has been to treat it medically, driven by the belief that it would resolve upon resolution of the underlying cause [7,24-26]. Nonetheless, recent evidence from several studies suggests not only that significant TR impacts negatively patient prognosis (Fig. 2) [7,15,22], but also that it does so more and more for every increase in grade of severity $[23,27]$. This might be well explained by the fact that chronic RV volume overload due to self-perpetuating significant TR may result in progressive RV dilation and remodeling, which in turn might then lead to progressive irreversible RV myocardial damage and symptomatic right heart failure [28]. In advanced stages, low cardiac output might not only be due to the relevant amount of regurgitant flow at expense of forward flow, but also due to the inability to proportionally increase cardiac output to metabolic needs related to increased left heart pressures caused by diastolic ventricular interaction and pericardial restraint [29,30]. In the end, such natural history suggests a non-marginal role of TR in disease, especially upon the onset of the vicious cycle encompassing RV myocardial damage and remodeling. 


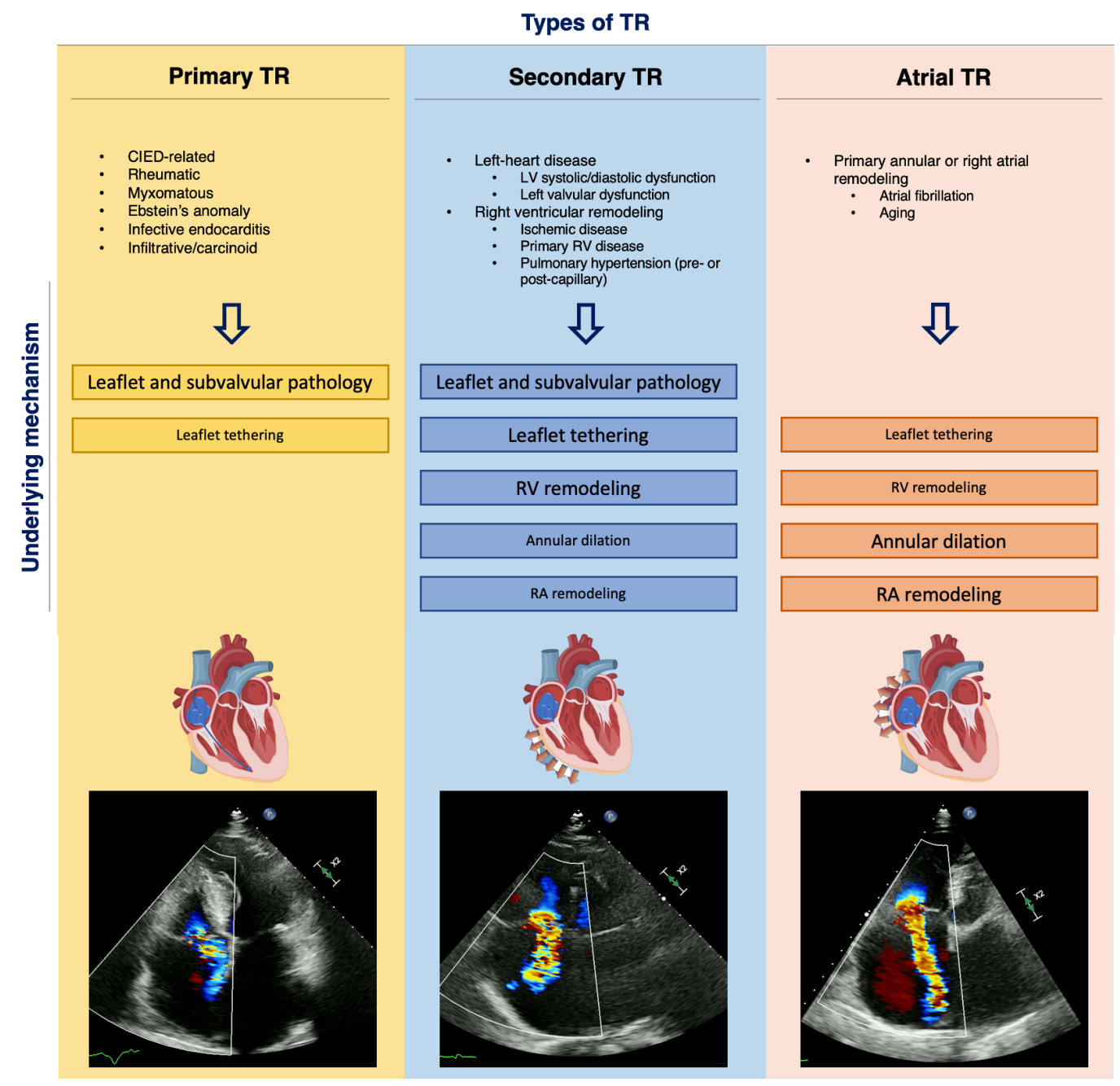

Fig. 1. Classification of types of tricuspid regurgitation and related mechanisms. The three main types of TR are associated with different mechanisms of disease, which may overlap. CIED, cardiovascular implantable electronic device; LV, left ventricle; RA, right atrium; RV, right ventricle.

\section{Innocent bystander or covert foe-does severity of regurgitation matter?}

Of note, whether TR predicts mortality independently from left-sided valve disease, pulmonary hypertension, RV dysfunction and other comorbidities has only recently been investigated in a more punctual and thorough manner [7, 15,31]. Not surprisingly, the severity of disease does play a central role:

- Severe TR is associated with increased risk of mortality when compared with patients with no TR in retrospective registries, with reported overall mortality rates at 1 -year of up to almost $50 \%$ vs. $8 \%$, respectively [7,23]. Of note, the association of severe TR with increased overall mortality persists when adjusting also for RV function, with more than $40 \%$ increase in events as compared with patients with no TR [23].

- Moderate TR also yields a 15\% increase in mortality when compared with no or minimal TR [23]. Patients with moderate or severe primary or secondary TR from a comprehensive, although heterogeneous, large-scale metaanalysis including more than 30,000 patients [27] had double the risk of overall mortality when compared with no or minimal TR. Such results might allow inference on the generalizability of the dreadful prognostic impact of TR in different settings, irrespective of the underlying etiology $[2,7,22,32-34]$.

- Mild TR has been linked to increased mortality as well [27]. This might be related to the progressive nature of TR, and the fact that TR progression is paralleled by worse outcomes [35-37]. Also, the rapidity of significant TR development impacts all-cause mortality $[33,36]$. Presence of pacemaker or defibrillator leads, surgical left-sided valvular treatment, elevated pulmonary pressures, increase of RV sphericity, tricuspid annular dilatation and increased TV tenting area predict fast development of significant TR [35], and could reveal useful for early identification of patients at risk and intervention before onset of irreversible right ventricular dysfunction. 
Table 1. Selected evidence on outcomes in patients with tricuspid regurgitation in different clinical settings.

\begin{tabular}{|c|c|c|c|c|c|}
\hline & Year, author & $\mathbf{N}$ & Population & $\begin{array}{l}\text { Follow-up } \\
\text { (years) }\end{array}$ & Main results \\
\hline \multirow[t]{6}{*}{ Mitral valve disease } & 2003, Henein et al. [9] & 42 & MVR for rheumatic disease & 5 & $50 \%$ vs. $100 \%$ survival in patients with severe vs. mild TR \\
\hline & 2004, Ruel et al. [10] & 708 & MVR & 5 & Increased risk of mortality with persistent TR (HR 2.17) \\
\hline & 2009, Di Mauro et al. [11] & 165 & Mitral valve surgery for secondary MR & 5 & $\begin{array}{l}\text { Increased risk of mortality with moderate or more vs. mild or less } \\
\text { TR (HR 3.1) }\end{array}$ \\
\hline & 2014, Ohno et al. [12] & 146 & TEER for secondary MR & 1 & $\begin{array}{l}\text { Moderate/severe TR predicted death and re-hospitalization for heart } \\
\text { failure (HR 2.67) }\end{array}$ \\
\hline & 2020, Hahn et al. [13] & 599 & $\begin{array}{l}\text { Medical therapy alone or medical therapy and } \\
\text { TEER for secondary MR }\end{array}$ & 2 & $\begin{array}{l}\text { Increased risk of death and hospitalization for heart failure if mod- } \\
\text { erate or more TR treated with medical therapy alone (HR 1.74) }\end{array}$ \\
\hline & 2020, Essayagh et al. [14] & 5083 & Primary MR & 10 & $\begin{array}{l}\text { Increased adjusted risk of mortality with moderate (HR 1.40) and } \\
\text { severe TR (HR 2.10) }\end{array}$ \\
\hline \multirow[t]{5}{*}{ LV dysfunction } & 2002, Koelling et al. [15] & 1,421 & Left ventricular ejection fraction $35 \%$ or less & 1 & Increased adjusted risk of poor outcome with severe TR (HR 1.55) \\
\hline & 2012, Agricola et al. [16] & 373 & $\begin{array}{l}\text { Heart failure, left ventricular ejection fraction } \\
<50 \% \text { and mild or more secondary MR }\end{array}$ & 3 & $\begin{array}{l}\text { Moderate to severe functional TR independently predicted heart } \\
\text { failure (HR 1.4) and mortality (HR 1.6) }\end{array}$ \\
\hline & 2013, Neuhold et al. [17] & 576 & Congestive systolic heart failure & 6 & $\begin{array}{l}\text { Increased risk of death/heart transplantation/left ventricular-assist } \\
\text { device implantation in patients with TR (HR 2.07) }\end{array}$ \\
\hline & 2019, Benfari et al. [8] & 11,507 & $\begin{array}{l}\text { Heart failure stage } \mathrm{B}-\mathrm{C} \text {, left ventricular ejection } \\
\text { fraction }<50 \%\end{array}$ & 4 & $\begin{array}{l}\text { Increased adjusted risk of mortality with mild (HR 1.09), moderate } \\
\text { (HR 1.21) and severe TR (HR 1.57) vs. trivial TR }\end{array}$ \\
\hline & 2019, Bartko et al. [18] & 382 & Heart failure and reduced ejection fraction & 5 & $\begin{array}{l}0.9 \% \text { and } 1.3 \% \text { increased risk of mortality per } 1 \mathrm{~mm}^{2} \text { increase in } \\
\text { EROA and } 1 \mathrm{~mL} \text { increase in regurgitant volume, respectively }\end{array}$ \\
\hline Aortic valve disease & 2021, Tomii et al. [19] & 2,008 & TAVI & 1 & $\begin{array}{l}\text { Excess risk of mortality with severe }(90 \%) \text { and massive TR }(117 \%) \\
\text { after TAVI }\end{array}$ \\
\hline \multirow[t]{5}{*}{ Other settings } & 2004, Messika-Zeitoun et al. [20] & 60 & TR due to flail leaflets & 10 & $\begin{array}{l}\text { Excess risk of mortality ( } 39 \%) \text { with severe TR when compared to } \\
\text { matched United States population }\end{array}$ \\
\hline & 2014, Hoke et al. [21] & 239 & CIED-related TR & 5 & $\begin{array}{l}\text { Increased risk of mortality and heart failure-related events with sig- } \\
\text { nificant TR (HR 1.69) }\end{array}$ \\
\hline & 2014, Topilsky et al. [22] & 353 & Atrial TR & 6 & $\begin{array}{l}\text { Increased risk of mortality with qualitatively and quantitatively } \\
\text { measured severe TR (HR } 1.78 \text { and } 2.67 \text {, respectively) }\end{array}$ \\
\hline & 2019, Topilsky et al. [2] & 417 & $\begin{array}{l}\text { Moderate or more } \mathrm{TR} \text { of various etiologies } \\
(8.1 \% \text { atrial TR })\end{array}$ & Up to 15 & Increased risk of mortality with atrial TR (HR 1.68) \\
\hline & 2020, Chorin et al. [23] & 33,305 & TR of various etiologies & 3 & $\begin{array}{l}\text { Increased risk of mortality with moderate (1.15) and severe TR } \\
(1.43) \text { vs. no or minimal TR }\end{array}$ \\
\hline
\end{tabular}

EROA, effective regurgitant orifice area; HR, hazard ratio; MR, mitral regurgitation; MVR, mitral valve replacement TEER, transcatheter edge-to-edge repair; TR, tricuspid 


\section{Tricuspid regurgitation in mitral valve disease}

TR is frequently present in patients with primary and secondary mitral valve disease, and may occur also late after mitral valve intervention [15,38-42]. For this reason, aggressive correction with tricuspid annuloplasty at the time of mitral valve surgery is actually encouraged.

\subsection{Tricuspid regurgitation in rheumatic primary mitral valve disease}

More than $30 \%$ of patients with mitral stenosis have at least moderate TR $[43,44]$, which is associated with higher New York Heart Association (NYHA) functional class than mild or no TR at long term follow-up [44]. Also, patients with pre-procedural severe TR among those undergoing balloon mitral valvotomy not only present more advanced mitral valve disease, higher pulmonary vascular resistance and smaller increase in mitral valve area after valvotomy, but also they have poorer outcome in terms of overall survival, heart failure episodes and need for repeat intervention or mitral valve replacement (MVR) [45]. The incidence of moderate or severe late TR in rheumatic patients is up to $37 \%$ and $68 \%$, respectively. In these cases, TR is often diagnosed years after MVR, ten on average [46,47]. The pathophysiological basis for such condition is poorly defined [48], nonetheless significant TR is associated with higher NYHA functional class III or IV, heart failure episodes and all-cause death at 5 years $[9,10]$.

\subsection{Tricuspid regurgitation in non-rheumatic primary mitral valve disease}

TR is common among patients undergoing mitral valve surgery for primary mitral regurgitation (MR) [2,14]. When severe, TR is often addressed during the index procedure, but the operative management of mild or moderate TR is widely debated. If not corrected at the time of left-sided cardiac surgery, TR may progress in a quarter of patients, especially in presence of annular dilation of 40 $\mathrm{mm}$ (or $21 \mathrm{~mm} / \mathrm{m}^{2}$ ) or more in diameter, leaflet tethering, transvalvular leads, RV dysfunction, pulmonary hypertension and atrial fibrillation [49], and finally jeopardize late survival and functional outcomes [50,51]. It is important to underline that patients with severe TR after mitral valve surgery undergoing isolated TV surgery usually have high perioperative mortality (10\%) and poor late survival [5255], so that TV repair at index procedure may limit disease progression and eventually provide long-term clinical benefit [11].

\subsection{Tricuspid regurgitation in secondary mitral valve disease}

When focusing on patients with secondary MR undergoing mitral valve surgery, the impact of at least moderate functional TR on outcomes is substantial. Indeed, affected patients presented one half the chance to be alive at 5 years when compared with patients with less than moderate TR [11].

Thus, The prognostic role of tricuspid regurgitation (TR) associated with organic left-sided valvular heart disease is well known.

More recent reports addressed the role of TR in patients undergoing transcatheter edge-to-edge repair (TEER) treatment of secondary MR. Although TEER leads to improvement in MR, TR and NYHA functional class irrespective of baseline TR, NYHA functional class $>2$ was more common in patients with moderate/severe TR, suggesting that significant TR limits mid-term benefits of TEER in patients affected by secondary MR. Of note, moderate/severe TR independently predicted death and re-hospitalization for heart failure at 1 year [12]. In a recent analysis [13], patients with at least moderate TR presented worse clinical and echocardiographic characteristics than those with no or mild TR, and had higher rate of the composite of death or hospitalization for heart failure at 2 years when treated with medical therapy alone, while this was not the case following TEER with MitraClip.

Finally, although TR after mitral valve surgery has mostly been reported in patients with primary mitral valve disease, it may present also in patients after surgical repair of MR due to dilated ischemic or non-ischemic cardiomyopathy [56-58].

\section{Tricuspid regurgitation in aortic valve disease}

Secondary TR is often found in conjunction with aortic stenosis, especially when low-flow, low-gradient, and it is associated with increased risk of mortality [59]. The reported incidence of TR in patients candidate to surgical aortic valve replacement or transcatheter aortic valve implantation (TAVI) is of $11 \%$ [60]. TR does not improve up to 4 years after intervention in almost half of patients undergoing aortic valve replacement [61], and post-operative progression is observed in a quarter of patients [60]. In a recent analysis conducted on patients undergoing TAVI, more than $50 \%$ of patients experienced TR reduction after the procedure, whereas in little less than $10 \%$ of cases TR worsened [19]. Such evidence may lead to speculate that, in order to avoid late TR, a concomitant TV procedure might be considered in selected patients.

The impact of TR in patients undergoing TAVI has also been recently addressed: severe and massive TR postTAVI is associated with a 2-fold increased risk of 1-year all-cause death, while this is not the case for baseline TR severity [19].

\section{Tricuspid regurgitation in left ventricle systolic dysfunction}

Notwithstanding the pathophysiological mechanisms described above by which TR can negatively affect patient outcomes, its independent association to poor prognosis in 


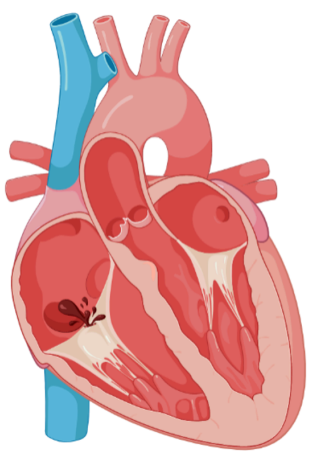

Abnormal coaptation Annular dilation and RV remodelling

No symptoms Responsive to medical therapy

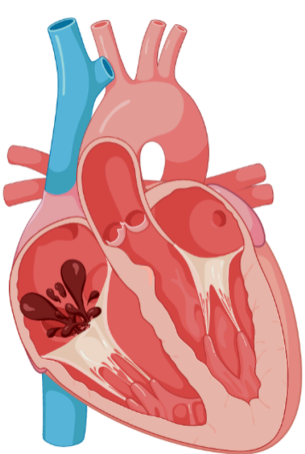

Maladaptive RV remodelling RV dysfunction

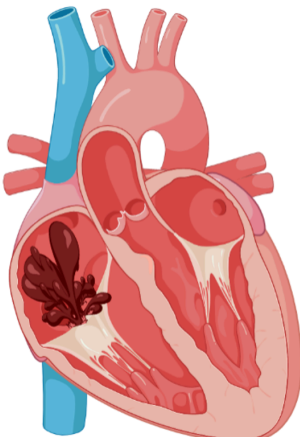

Fluid retention

Liver and kidney dysfunction Low cardiac output

Need for high dose diuretics Recurrent admission for RHF Prohibitive surgical risk Consider surgical (risk of perioperative RV dysfunction) or percutaneous approach (still limited evidence)

Fig. 2. The role of tricuspid regurgitation in disease. Incremental impact on survival is witnessed with worsening of the grade of TR. Mechanisms of disease and therapeutic considerations according to severity of TR are also depicted. RHF, right heart failure; RV, right ventricle; TR, tricuspid regurgitation.

the subset of patients with left ventricle (LV) systolic dysfunction has been subject of debate as well. In this population, functional TR has been consistently associated with more dyspnea, older age, renal dysfunction, lower cardiac output, lower LV ejection fraction, MR, atrial fibrillation, and pulmonary hypertension [58]. Nonetheless, evidence of TR as a prognostic interventional target rather than mere indicator of worse prognosis in patients with heart failure has been accruing [62].

Early reports revealed a strong impact of TR on clinical outcome [15], and this is true also when focusing on patients with severe heart failure secondary to idiopathicdilated cardiomyopathy or to ischemic cardiomyopathy [42]. When compared to trivial TR, different degrees of TR jeopardize outcomes to different levels in patients with secondary TR and heart failure with reduced LV ejection fraction. In particular, $9 \%, 21 \%$ and $57 \%$ increase in mortality is described at long-term follow-up for patients with mild, moderate and severe TR, independently of LV systolic dysfunction and pulmonary hypertension [8].

\subsection{Does severity of heart failure matter?}

Of note, the prognostic role of TR might depend upon the degree of heart failure. Indeed, when stratifying patients according to mildly or moderately vs. severely impaired LV function, TR is significantly associated to the risk of death/heart transplantation/LV assist device implantation in the former group, not in the latter [17]. Also, when stratifying patients according to N-terminal-pro-B-type natriuretic peptide concentrations, TR is related with outcome in patients with concentrations below the median, not those with values above it [63]. The impact of TR in advanced heart failure might thus be limited, and might be considered merely a marker of advanced disease, and, as such, not an attractive therapeutic target. On the other hand, treatment of severe TR might have a favorable impact on outcome in patients with less severe HF.

\subsection{Does quantification of tricuspid regurgitation severity matter?}

Earlier studies addressing significance of TR in chronic heart failure used qualitative measures of TR grading, but assessment of secondary TR severity by quantitative measures might be useful to unravel its natural history [18]. Quantitative metrics of TR severity were indeed found to be consistently associated with mortality; namely, an increase in risk of mortality of $0.9 \%$ and $1.3 \%$ were observed per $1 \mathrm{~mm}^{2}$ increase in effective regurgitant orifice area (EROA) and per $1 \mathrm{~mL}$ increase in regurgitant volume, respectively. Severe functional TR (defined as EROA $>40 \mathrm{~mm}^{2}$ ) yields an increased risk of mortality and cardiac events of $80 \%$ and $120 \%$, respectively [32]. 
Even more striking, thresholds for moderate TR as per current guidelines, that is $\geq 20 \mathrm{~mm}^{2}$ of EROA, $>5 \mathrm{~mm}$ of vena contracta and $>20 \mathrm{~mL}$ of regurgitant volume, are actually associated with increased mortality [63]. Moderate to severe functional TR increased the risk of heart failure and overall mortality also in patients with at least mild secondary MR. In particular, patients with no, mild, moderate and severe functional TR had a survival free of all-cause mortality at 6 years of $69 \%, 67 \%, 51 \%$ and $40 \%$, respectively [16].

Overall, the evidence supports an important prognostic role for TR in patients with LV systolic dysfunction and calls for a paradigm shift in evaluation and therapeutic approach of patients with LV systolic dysfunction and secondary TR. Interventions aiming at TR treatment should be investigated also in this population, and the definition of the appropriate timing for intervention will be of paramount importance. In this setting, awareness not only of the dynamic nature of TR, but also of the risk of worsening pulmonary hypertension, right ventricular remodeling and mortality associated with non-severe TR progression is key $[36,64]$.

\section{Tricuspid regurgitation in other settings}

As already introduced, additional morphologic types of TR can be recognized, including primary and atrial TR [65], and it is important to recognize that although they often have a separate course of disease, they might coexist in the settings described above as well [66]. As already introduced, the main cause of TR is mitral or aortic valve disease, in more than $50 \%$ of TR cases [1]. On the other hand, a primary etiology is present in $7.4 \%$ of TR patients, with cardiovascular implantable electronic device (CIED)-related TR being the most common cause $(66 \%)$, while atrial TR is present in $17 \%$ of non-primary cases $[1,65]$.

\subsection{Primary tricuspid regurgitation}

Patients with severe primary TR due to any congenital or organic cause, irrespective of LV function, left valvular function or pulmonary pressure, demonstrate a trend towards worse prognosis than those with no or trace TR [23].

The mechanism behind CIED-related TR, the most common cause of primary TR, is usually impingement of leaflet, subvalvular apparatus or papillary muscles, but leaflet perforation, fibrosis, thrombosis and endocarditis may occur as well. In this subgroup of patients, worse longterm survival and increased risk of heart failure-related events are reported when patients with significant TR are compared to those without significant TR, especially when focusing on patients with baseline LV ejection fraction $<40 \%$ [21].

TV leaflet flail is often due to trauma, and leads to TR of mostly severe grade. Excess mortality is present in patients with TR due to flail leaflets, and excess tricuspidrelated events accrue also in those asymptomatic at presentation. Moreover, severe dilation of right-sided cham- bers is an independent predictor of poor outcomes in such patients, so that early intervention before irreversible right heart chamber remodeling and dysfunction should be considered [20].

\subsection{Atrial tricuspid regurgitation}

Atrial TR is defined as such when holosystolic and functional, with no likely pulmonary hypertension, no overt cause and no previous valve surgery [27]. It is important to note that outcomes do differ when analyzing TR patients according to etiology. Indeed, atrial TR, even though associated with excess mortality, was observed to have less impact on yearly mortality, atrial fibrillation or heart failure hospitalization rates when compared to secondary or even primary disease [2]. Nonetheless, the reported 10-year survival rate of patients with an effective regurgitant orifice $\geq 40 \mathrm{~mm}^{2}$ is $38 \%$, and severe TR predicts higher mortality when graded both qualitatively and quantitatively, regardless of RV size or function, pulmonary pressures or relevant comorbidities [27,36]. The surgical benefit in patients with significant atrial TR was also investigated, and a trend towards benefit in survival rate was observed when comparing patients undergoing TV surgery and patients managed medically [67].

\section{Reappraisal of adequate indication and timing for treatment of tricuspid regurgitation}

The proposed algorithm in the latest American College of Cardiology/American Heart Association guidelines [68] supports TV surgery with Class I indication only at the time of left-sided valve surgery. On the other hand, only Class IIa and IIb indications exist for treatment of TR in other clinical settings, and these are based upon evaluation not only of TR severity and etiology, but also of symptoms, severity of pulmonary hypertension and RV size and function. The Class I indication to proceed to surgery for severe symptomatic isolated TR in absence of severe RV dysfunction and Class IIa indication for surgery in severe secondary TR either symptomatic or progressive RV dilation reported in the latest European Society of Cardiology guidelines might stem from the recent evidence of improved outcomes after isolated TV surgery with careful patient selection and current perioperative management [69].

In addition, the latest European guidelines report transcatheter treatment of symptomatic severe secondary TR in inoperable patients with a Class IIb indication (Table 2) [70]. Thus, upon re-evaluation of correct indication and timing of TR intervention, different factors need to be taken into account.

\subsection{Tricuspid regurgitation grading}

Despite the evidence that quantitative grading of TR is a strong independent predictor of clinical outcomes, even superior to qualitative assessment [22], only one single 


\begin{tabular}{|c|c|c|}
\hline & $\begin{array}{l}\text { Class of } \\
\text { recommendation }\end{array}$ & $\begin{array}{l}\text { Level of } \\
\text { evidence }\end{array}$ \\
\hline \multicolumn{3}{|l|}{ AHA/ACC 2020 Guidelines } \\
\hline \multicolumn{3}{|l|}{ Primary TR } \\
\hline Surgery for severe TR at the time of left-sided valve surgery & I & $\mathrm{B}$ \\
\hline Surgery for symptoms caused by severe TR not responsive to medical therapy & IIa & $\mathrm{B}$ \\
\hline Surgery for asymptomatic severe TR and progressive right ventricular dilatation and dysfunction & $\mathrm{IIb}$ & $\mathrm{C}$ \\
\hline \multicolumn{3}{|l|}{ Secondary TR } \\
\hline Surgery for severe TR at the time of left-sided valve surgery & I & $\mathrm{B}$ \\
\hline $\begin{array}{l}\text { Surgery for progressive TR at the time of left-sided valve surgery with either a dilated annulus } \\
\text { ( } \geq 40 \mathrm{~mm} \text { or } \geq 21 \mathrm{~mm} / \mathrm{m}^{2} \text { ) or evidence of previous right heart failure }\end{array}$ & IIa & $\mathrm{B}$ \\
\hline $\begin{array}{l}\text { Surgery for symptoms caused by severe TR attributable to annular dilation (in the absence of } \\
\text { pulmonary hypertension or left-sided dysfunction) not responsive to medical therapy }\end{array}$ & IIa & $\mathrm{B}$ \\
\hline $\begin{array}{l}\text { Surgery for persistent symptoms caused by isolated severe TR after previous left-sided valve } \\
\text { surgery in absence of severe pulmonary hypertension or right ventricular dysfunction }\end{array}$ & $\mathrm{IIb}$ & $\mathrm{B}$ \\
\hline \multicolumn{3}{|l|}{ ESC/EACTS 2021 guidelines } \\
\hline \multicolumn{3}{|l|}{ Primary TR } \\
\hline Surgery for severe TR at the time of left-sided valve surgery & I & $\mathrm{C}$ \\
\hline Surgery for severe symptomatic isolated TR without severe right ventricular dysfunction & I & $\mathrm{C}$ \\
\hline Surgery for moderate TR at the time of left-sided valve surgery & IIa & $\mathrm{C}$ \\
\hline $\begin{array}{l}\text { Surgery for asymptomatic or mildly symptomatic isolated severe TR and right ventricular di- } \\
\text { latation, if appropriate for surgery }\end{array}$ & IIa & $\mathrm{C}$ \\
\hline \multicolumn{3}{|l|}{ Secondary TR } \\
\hline Surgery for severe TR at the time of left-sided valve surgery & I & $\mathrm{B}$ \\
\hline $\begin{array}{l}\text { Surgery for mild or moderate TR with dilated annulus }\left(\geq 40 \mathrm{~mm} \text { or } \geq 21 \mathrm{~mm} / \mathrm{m}^{2}\right) \text { at the time of } \\
\text { left-sided valve surgery }\end{array}$ & IIa & $\mathrm{B}$ \\
\hline $\begin{array}{l}\text { Surgery for severe TR with or without previous left-sided valve surgery with symptoms or pro- } \\
\text { gressive right ventricular dilatation, in absence of severe right or left ventricular dysfunction } \\
\text { and severe pulmonary vascular disease/hypertension }\end{array}$ & IIa & B \\
\hline $\begin{array}{l}\text { Transcatheter treatment for symptomatic severe TR in inoperable patients at a Heart Valve Cen- } \\
\text { tre with expertise in the treatment of tricuspid valve disease }\end{array}$ & IIb & $\mathrm{C}$ \\
\hline
\end{tabular}

study reported results according to such grading in a recent meta-analysis of 70 studies [27]. Given the possible underestimation of severity of disease with biplanar measurement of vena contracta [71], focus moved upon the utility of proximal isovelocity hemispheric surface area (PISA) for risk stratification [22]. Nonetheless, it is important to recognize that PISA might be underestimating TR severity as well [71-73], which might explain the sustained increase in mortality associated with EROA $>20 \mathrm{~mm}^{2}$ and regurgitant volume $>20 \mathrm{~mL}$ [18]. An additional issue is the extended grading scheme of TR [74] that was recently proposed, which addresses the unmet need of classifying patients currently undergoing percutaneous treatment in clinical trials, who often suffer from a degree of TR greater than merely severe, be it for the long symptoms tolerance or for the missed acknowledgment of the disease. In this context, many studies investigated the role of using additional cutoffs for vena contracta width and EROA, and consistently showed worse prognosis in patients classified as having ei- ther massive or torrential TR [72-75].

\subsection{The right ventricle and pulmonary artery coupling}

Quantification of RV size and function [75-77], of severity of pulmonary hypertension and of the relationship between RV and pulmonary artery [78,79] should be integral to the evaluation of TR. Ventriculo-arterial coupling represents the ability of the RV to balance its contractility with the afterload due to the pulmonary arterial vascular bed. Its reference method of assessment is pressure-volume loop analysis, but several non-invasive surrogated combining indices of RV function or chamber size with a metric of load have been proposed [78,80]. Recently, abnormal ventriculo-arterial coupling, defined as RV free wall longitudinal strain/RV systolic pressure $\leq 0.5 \% / \mathrm{mmHg}$, predicted all-cause mortality in patients with severe secondary MR enrolled in the Cardiovascular Outcomes Assessment of the MitraClip Percutaneous Therapy for Heart Failure Patients With Functional Mitral Regurgitation (COAPT) 
trial [81]. Right ventricular systolic pressure is also often measured non-invasively by estimation via TR velocity, but some limitations need to be ascertained, especially when encountering patients with higher degrees of severity of TR. Indeed, discordance of pulmonary hypertension estimation via invasive and non-invasive measurement was a marker of more advanced valvular and ventricular disease, and should be taken into account when predicting procedural success and long-term outcomes [82]. It is thus important to keep in mind that invasive measurements yielding often underestimated hemodynamic information, including the type and eventual reversibility of pulmonary hypertension, might shed light onto the optimal TR population to be treated.

\subsection{Implications for intervention}

The first major implication for intervention is that TR should be treated during index left-sided valve surgery, especially if the latter is of rheumatic origin. Given the wide variation in adoption of concomitant TV and mitral valve surgery $[8,49,83,84]$, evidence not only of the absence of increased risk-adjusted mortality irrespective of the grade of TR, but actually of the lower risk of cardiac-related mortality and improved echocardiographic TR outcomes after concomitant TV repair at the time of left-sided valve surgery is of particular importance $[50,51,83]$.

Similarly, prevention of long-term progression of functional TR by adopting a more aggressive strategy involving concomitant TR treatment upon mitral valve surgery should be acknowledged, especially when considering optimal timing for intervention $[70,85]$.

The second major implication for intervention is that, notwithstanding the potential for positively affecting the natural history of TR, isolated TV surgery has long been paralleled by high risk of mortality. Unfortunately, it has been difficult to generate prospective randomized evidence to demonstrate whether isolated TV surgery for TR has a positive prognostic effect, mainly due to a vicious cycle deriving from high reported mortality (9-10\%), in-hospital complications (31\%), poor late survival and no significant improvement in functional capacity after TV surgery $[9,10,47,48]$, yielding reluctancy of cardiologists to refer patients to surgery unless extremely symptomatic. Indeed, given the good response of TR to diuretic therapy, referral usually occurred late in the course of the disease concomitantly with onset of RV dysfunction. Earlier referral of TV surgery before RV function compromise might yield better outcomes [53], and, although not thoroughly investigated yet, recent studies reported promising results [69,86,87].

Finally, pre-operative clinical and echocardiographic features affect outcomes more than etiology or mechanism of TR [88], so that scoring systems to predict mortality after TV surgery might reveal useful to properly select patients to intervene upon and guide clinical decision-making [85]. Taking into consideration the lessons learned in the surgical context $[51,86,87]$, the introduction of transcatheter tricuspid valve interventions might offer patients a less invasive and safer procedure [6]. Appropriate patient selection and timing of intervention will be of paramount importance in order to impact positively the history of disease and avoid futility in patients at a too advanced disease stage.

\section{Conclusions}

There is a growing body of evidence today that significant TR is not merely a bystander in the different disease processes in which it is involved, but rather is an active player in the pathophysiology and evolution of disease, impacting negatively on outcomes. Moreover, recent advances in diagnostic evaluation and risk stratification will allow us to more appropriately identify the adequate population to treat and the proper timing of intervention. Thus, we believe that the synergistic effect of medical, surgical and, more recently, percutaneous treatment of TR has the potential to positively affect clinical outcomes in this large population of patients, and testing of this concept in properly designed trials is eagerly awaited.

\begin{abstract}
Abbreviations
CIED, cardiovascular implantable electronic device; EROA, effective regurgitant orifice area; HR, hazard ratio; LV, left ventricular; MR, mitral regurgitation; MVR, mitral valve replacement; NYHA, New York Heart Association; PISA, proximal isovelocity surface area; RA, right atrium; RV, right ventricle; TAVI, transcatheter aortic valve implantation; TEER, transcatheter edge-to-edge repair; TR, tricuspid regurgitation; TV, tricuspid valve.
\end{abstract}

\section{Author contributions}

PPL and AM contributed to conception of the review. PPL, AM and MC contributed to design of the article. PPL wrote the first draft of the manuscript. MC wrote sections of the manuscript. DR, MP, LM, BP, FL, GS, DP, BR, AC, $\mathrm{AL}$ contributed to editorial changes and manuscript revision. PPL, MC, DR, MP, LM, BP, FL, GS, DP, BR, AC, $\mathrm{AL}$ and $\mathrm{AM}$ read and approved the submitted version.

\section{Ethics approval and consent to participate}

Not applicable.

\section{Acknowledgment}

We would like to express our gratitude to all those who helped us during the writing of this manuscript.

\section{Funding}

This research received no external funding.

\section{Conflict of interest}

The authors declare no conflict of interest. Azeem Latib and Antonio Mangieri are serving as Guest editors 
of this journal. We declare that Azeem Latib and Antonio Mangieri had no involvement in the peer review of this article and have no access to information regarding its peer review. Full responsibility for the editorial process for this article was delegated to Alexander Lauten.

\section{References}

[1] Singh JP, Evans JC, Levy D, Larson MG, Freed LA, Fuller DL, et al. Prevalence and clinical determinants of mitral, tricuspid, and aortic regurgitation (The Framingham Heart Study). The American Journal of Cardiology. 1999; 83: 897-902.

[2] Topilsky Y, Maltais S, Medina Inojosa J, Oguz D, Michelena $\mathrm{H}$, Maalouf $\mathrm{J}$, et al. Burden of Tricuspid Regurgitation in Patients Diagnosed in the Community Setting. JACC: Cardiovascular Imaging. 2019; 12: 433-442.

[3] d'Arcy JL, Coffey S, Loudon MA, Kennedy A, Pearson-Stuttard $\mathrm{J}$, Birks $\mathrm{J}$, et al. Large-scale community echocardiographic screening reveals a major burden of undiagnosed valvular heart disease in older people: the OxVALVE Population Cohort Study. European Heart Journal. 2016; 37: 3515-3522.

[4] Prihadi EA, Delgado V, Leon MB, Enriquez-Sarano M, Topilsky Y, Bax JJ. Morphologic Types of Tricuspid Regurgitation: Characteristics and Prognostic Implications. JACC: Cardiovascular Imaging. 2019; 12: 491-499.

[5] Matta M, Layoun H, Abou Hassan OK, Rodriguez L, Schoenhagen $\mathrm{P}$, Kanj M, et al. Mechanistic Insights into Significant Atrial Functional Tricuspid Regurgitation. JACC: Cardiovascular Imaging. 2021; 14: 2049-2050.

[6] Praz F, Muraru D, Kreidel F, Lurz P, Hahn RT, Delgado V, et al. Transcatheter treatment for tricuspid valve disease. EuroIntervention. 2021; 17: 791-808.

[7] Nath J, Foster E, Heidenreich PA. Impact of tricuspid regurgitation on long-term survival. Journal of the American College of Cardiology. 2004; 43: 405-409.

[8] Benfari G, Antoine C, Miller WL, Thapa P, Topilsky Y, Rossi A, et al. Excess Mortality Associated with Functional Tricuspid Regurgitation Complicating Heart Failure with Reduced Ejection Fraction. Circulation. 2019; 140: 196-206.

[9] Henein MY, O'Sullivan CA, Li W, Sheppard M, Ho Y, Pepper $\mathrm{J}$, et al. Evidence for rheumatic valve disease in patients with severe tricuspid regurgitation long after mitral valve surgery: the role of $3 \mathrm{D}$ echo reconstruction. The Journal of Heart Valve Disease. $2003 ; 12$ : 566-572.

[10] Ruel M, Rubens FD, Masters RG, Pipe AL, Bédard P, Mesana TG. Late incidence and predictors of persistent or recurrent heart failure in patients with mitral prosthetic valves. The Journal of Thoracic and Cardiovascular Surgery. 2004; 128: 278-283.

[11] Di Mauro M, Bivona A, Iacò AL, Contini M, Gagliardi M, Varone E, et al. Mitral valve surgery for functional mitral regurgitation: prognostic role of tricuspid regurgitation. European Journal of Cardio-Thoracic Surgery. 2009; 35: 635-640.

[12] Ohno Y, Attizzani GF, Capodanno D, Cannata S, Dipasqua F, Immé $\mathrm{S}$, et al. Association of tricuspid regurgitation with clinical and echocardiographic outcomes after percutaneous mitral valve repair with the MitraClip System: 30-day and 12-month follow-up from the GRASP Registry. European Heart Journal Cardiovascular Imaging. 2014; 15: 1246-1255.

[13] Hahn RT, Asch F, Weissman NJ, Grayburn P, Kar S, Lim S, et al. Impact of Tricuspid Regurgitation on Clinical Outcomes: The COAPT Trial. Journal of the American College of Cardiology. 2020; 76: 1305-1314.

[14] Essayagh B, Antoine C, Benfari G, Maalouf J, Michelena HI, Crestanello JA, et al. Functional tricuspid regurgitation of degenerative mitral valve disease: a crucial determinant of survival. European Heart Journal. 2020; 41: 1918-1929.
[15] Koelling TM, Aaronson KD, Cody RJ, Bach DS, Armstrong WF. Prognostic significance of mitral regurgitation and tricuspid regurgitation in patients with left ventricular systolic dysfunction. American Heart Journal. 2002; 144: 524-529.

[16] Agricola E, Stella S, Gullace M, Ingallina G, D’Amato R, Slavich $\mathrm{M}$, et al. Impact of functional tricuspid regurgitation on heart failure and death in patients with functional mitral regurgitation and left ventricular dysfunction. European Journal of Heart Failure. 2012; 14: 902-908.

[17] Neuhold S, Huelsmann M, Pernicka E, Graf A, Bonderman D, Adlbrecht $\mathrm{C}$, et al. Impact of tricuspid regurgitation on survival in patients with chronic heart failure: unexpected findings of a long-term observational study. European Heart Journal. 2013; 34: 844-852.

[18] Bartko PE, Arfsten H, Frey MK, Heitzinger G, Pavo N, Cho A, et al. Natural History of Functional Tricuspid Regurgitation: Implications of Quantitative Doppler Assessment. JACC: Cardiovascular Imaging. 2019; 12: 389-397.

[19] Tomii D, Okuno T, Praz F, Heg D, Wild MG, Lanz J, et al. Potential Candidates for Transcatheter Tricuspid Valve Intervention after Transcatheter Aortic Valve Replacement: Predictors and Prognosis. JACC: Cardiovascular Interventions. 2021; 14: 2246-2256.

[20] Messika-Zeitoun D, Thomson H, Bellamy M, Scott C, Tribouilloy C, Dearani J, et al. Medical and surgical outcome of tricuspid regurgitation caused by flail leaflets. The Journal of Thoracic and Cardiovascular Surgery. 2004; 128: 296-302.

[21] Höke U, Auger D, Thijssen J, Wolterbeek R, van der Velde ET, Holman ER, et al. Significant lead-induced tricuspid regurgitation is associated with poor prognosis at long-term follow-up. Heart. 2014; 100: 960-968.

[22] Topilsky Y, Nkomo VT, Vatury O, Michelena HI, Letourneau T, Suri RM, et al. Clinical outcome of isolated tricuspid regurgitation. JACC: Cardiovascular Imaging. 2014; 7: 1185-1194.

[23] Chorin E, Rozenbaum Z, Topilsky Y, Konigstein M, Ziv-Baran $\mathrm{T}$, Richert E, et al. Tricuspid regurgitation and long-term clinical outcomes. European Heart Journal Cardiovascular Imaging. 2020; 21: 157-165.

[24] BRAUNWALD NS, ROSS J, MORROW AG. Conservative Management of Tricuspid Regurgitation in Patients Undergoing Mitral Valve Replacement. Circulation. 1967; 35: I63-I69.

[25] Arbulu A, Holmes RJ, Asfaw I. Tricuspid valvulectomy without replacement. Twenty years' experience. The Journal of Thoracic and Cardiovascular Surgery. 1992; 102: 917-922.

[26] Mangieri A, Montalto C, Pagnesi M, Jabbour RJ, Rodés-Cabau J, Moat N, et al. Mechanism and Implications of the Tricuspid Regurgitation: from the Pathophysiology to the Current and Future Therapeutic Options. Circulation Cardiovascular Interventions. 2017; 10: e005043.

[27] Wang N, Fulcher J, Abeysuriya N, McGrady M, Wilcox I, Celermajer D, et al. Tricuspid regurgitation is associated with increased mortality independent of pulmonary pressures and right heart failure: a systematic review and meta-analysis. European Heart Journal. 2019; 40: 476-484.

[28] Voelkel NF, Quaife RA, Leinwand LA, Barst RJ, McGoon MD, Meldrum DR, et al. Right ventricular function and failure: report of a National Heart, Lung, and Blood Institute working group on cellular and molecular mechanisms of right heart failure. Circulation. 2006; 114: 1883-1891.

[29] Andersen MJ, Nishimura RA, Borlaug BA. The hemodynamic basis of exercise intolerance in tricuspid regurgitation. Circulation Heart Failure. 2014; 7: 911-917.

[30] Fortuni F, Butcher SC, Dietz MF, van der Bijl P, Prihadi EA, De Ferrari GM, et al. Right Ventricular-Pulmonary Arterial Coupling in Secondary Tricuspid Regurgitation. The American Journal of Cardiology. 2021; 148: 138-145. 
[31] Behm CZ, Nath J, Foster E. Clinical correlates and mortality of hemodynamically significant tricuspid regurgitation. The Journal of Heart Valve Disease. 2004; 13: 784-789.

[32] Topilsky Y, Inojosa JM, Benfari G, Vaturi O, Maltais S, Michelena $\mathrm{H}$, et al. Clinical presentation and outcome of tricuspid regurgitation in patients with systolic dysfunction. European Heart Journal. 2018; 39: 3584-3592.

[33] Prihadi EA, van der Bijl P, Gursoy E, Abou R, Mara Vollema E, Hahn RT, et al. Development of significant tricuspid regurgitation over time and prognostic implications: new insights into natural history. European Heart Journal. 2018; 39: 3574-3581.

[34] Bar N, Schwartz LA, Biner S, Aviram G, Ingbir M, Nachmany I, et al. Clinical Outcome of Isolated Tricuspid Regurgitation in Patients with Preserved Left Ventricular Ejection Fraction and Pulmonary Hypertension. Journal of the American Society of Echocardiography. 2018; 31: 34-41.

[35] Shiran A, Najjar R, Adawi S, Aronson D. Risk factors for progression of functional tricuspid regurgitation. The American Journal of Cardiology. 2014; 113: 995-1000.

[36] Medvedofsky D, Aronson D, Gomberg-Maitland M, Thomeas $\mathrm{V}$, Rich S, Spencer K, et al. Tricuspid regurgitation progression and regression in pulmonary arterial hypertension: implications for right ventricular and tricuspid valve apparatus geometry and patients outcome. European Heart Journal Cardiovascular Imaging. 2017; 18: 86-94.

[37] Goldstone AB, Howard JL, Cohen JE, MacArthur JW, Atluri $\mathrm{P}$, Kirkpatrick JN, et al. Natural history of coexistent tricuspid regurgitation in patients with degenerative mitral valve disease: implications for future guidelines. The Journal of Thoracic and Cardiovascular Surgery. 2014; 148: 2802-2809.

[38] Shiran A, Sagie A. Tricuspid Regurgitation in Mitral Valve Disease. Journal of the American College of Cardiology. 2009; 53: 401-408.

[39] Dreyfus GD, Chan KMJ. Functional tricuspid regurgitation: a more complex entity than it appears. Heart. 2009; 95: 868-869.

[40] David TE. Functional tricuspid regurgitation: a perplexing problem. Journal of the American Society of Echocardiography. 2009; 22: 904-906.

[41] Chan V, Burwash IG, Lam B-, Auyeung T, Tran A, Mesana TG, et al. Clinical and Echocardiographic Impact of Functional Tricuspid Regurgitation Repair at the Time of Mitral Valve Replacement. The Annals of Thoracic Surgery. 2009; 88: 12091215.

[42] Hung J, Koelling T, Semigran MJ, Dec GW, Levine RA, Di Salvo TG. Usefulness of echocardiographic determined tricuspid regurgitation in predicting event-free survival in severe heart failure secondary to idiopathic-dilated cardiomyopathy or to ischemic cardiomyopathy. The American Journal of Cardiology. 1998; 82: 1301-1303.

[43] Sagie A, Freitas N, Chen MH, Marshall JE, Weyman AE, Levine RA. Echocardiographic assessment of mitral stenosis and its associated valvular lesions in 205 patients and lack of association with mitral valve prolapse. Journal of the American Society of Echocardiography. 1997; 10: 141-148.

[44] Boyaci A, Gokce V, Topaloglu S, Korkmaz S, Goksel S. Outcome of significant functional tricuspid regurgitation late after mitral valve replacement for predominant rheumatic mitral stenosis. Angiology. 2007; 58: 336-342.

[45] Sagie A, Schwammenthal E, Newell JB, Harrell L, Joziatis TB, Weyman AE, et al. Significant tricuspid regurgitation is a marker for adverse outcome in patients undergoing percutaneous ballon mitral valvuloplasty. Journal of the American College of Cardiology. 1994; 24: 696-702.

[46] Porter A, Shapira Y, Wurzel M, Sulkes J, Vaturi M, Adler Y, et al. Tricuspid regurgitation late after mitral valve replacement: clinical and echocardiographic evaluation. The Journal of Heart
Valve Disease. 1999; 8: 57-62.

[47] Izumi C, Iga K, Konishi T. Progression of isolated tricuspid regurgitation late after mitral valve surgery for rheumatic mitral valve disease. The Journal of Heart Valve Disease. 2002; 11: 353-356.

[48] Groves PH, Hall RJ. Late tricuspid regurgitation following mitral valve surgery. The Journal of Heart Valve Disease. 1992; 1: $80-86$.

[49] Chikwe J, Itagaki S, Anyanwu A, Adams DH. Impact of Concomitant Tricuspid Annuloplasty on Tricuspid Regurgitation, Right Ventricular Function, and Pulmonary Artery Hypertension after Repair of Mitral Valve Prolapse. Journal of the American College of Cardiology. 2015; 65: 1931-1938.

[50] Gammie JS, Chu MWA, Falk V, Overbey JR, Moskowitz AJ, Gillinov M, et al. Concomitant Tricuspid Repair in Patients with Degenerative Mitral Regurgitation. New England Journal of Medicine. 2022; 386: 327-339.

[51] Pagnesi M, Montalto C, Mangieri A, Agricola E, Puri R, Chiarito $\mathrm{M}$, et al. Tricuspid annuloplasty versus a conservative approach in patients with functional tricuspid regurgitation undergoing left-sided heart valve surgery: a study-level meta-analysis. International Journal of Cardiology. 2017; 240: 138-144.

[52] Mangoni AA, DiSalvo TG, Vlahakes GJ, Polanczyk CA, Fifer MA. Outcome following isolated tricuspid valve replacement. European Journal of Cardio-Thoracic Surgery. 2001; 19: 68-73.

[53] Kwon D, Park J, Chang H, Kim Y, Sohn D, Kim K, et al. Prediction of outcome in patients undergoing surgery for severe tricuspid regurgitation following mitral valve surgery and role of tricuspid annular systolic velocity. The American Journal of Cardiology. 2006; 98: 659-661.

[54] Jeganathan R, Armstrong S, Al-Alao B, David T. The risk and outcomes of reoperative tricuspid valve surgery. The Annals of Thoracic Surgery. 2013; 95: 119-124.

[55] Pfannmüller B, Moz M, Misfeld M, Borger MA, Funkat A, Garbade $\mathrm{J}$, et al. Isolated tricuspid valve surgery in patients with previous cardiac surgery. The Journal of Thoracic and Cardiovascular Surgery. 2013; 146: 841-847.

[56] De Bonis M, Lapenna E, Sorrentino F, La Canna G, Grimaldi A, Maisano F, et al. Evolution of tricuspid regurgitation after mitral valve repair for functional mitral regurgitation in dilated cardiomyopathy. European Journal of Cardio-Thoracic Surgery. 2008; 33: 600-606.

[57] Dreyfus GD, Corbi PJ, Chan KMJ, Bahrami T. Secondary Tricuspid Regurgitation or Dilatation: which should be the Criteria for Surgical Repair? The Annals of Thoracic Surgery. 2005; 79: 127-132.

[58] Matsunaga A, Duran CMG. Progression of Tricuspid Regurgitation after Repaired Functional Ischemic Mitral Regurgitation. Circulation. 2005; 112: I453-I457.

[59] Dahou A, Magne J, Clavel M, Capoulade R, Bartko PE, BerglerKlein J, et al. Tricuspid Regurgitation is Associated with Increased Risk of Mortality in Patients with Low-Flow LowGradient Aortic Stenosis and Reduced Ejection Fraction: Results of the Multicenter TOPAS Study (True or Pseudo-Severe Aortic Stenosis). JACC: Cardiovascular Interventions. 2015; 8: 588-596.

[60] Dumont C, Galli E, Oger E, Fournet M, Flecher E, Leclercq C, et al. Pre- and postoperative tricuspid regurgitation in patients with severe symptomatic aortic stenosis: importance of pre-operative tricuspid annulus diameter. European Heart Journal Cardiovascular Imaging. 2018; 19: 319-328.

[61] Jeong DS, Sung K, Kim WS, Lee YT, Yang J, Jun T, et al. Fate of functional tricuspid regurgitation in aortic stenosis after aortic valve replacement. The Journal of Thoracic and Cardiovascular Surgery. 2014; 148: 1328-1333. e1.

[62] Lancellotti P, Magne J. Tricuspid valve regurgitation in patients 
with heart failure: does it matter? European Heart Journal. 2013; 34: 799-801.

[63] Baumgartner H, Falk V, Bax JJ, De Bonis M, Hamm C, Holm PJ, et al. 2017 ESC/EACTS Guidelines for the management of valvular heart disease. European Heart Journal. 2017; 38: 2739 2791.

[64] Spinka G, Bartko PE, Heitzinger G, Prausmüller S, Pavo N, Frey MK, et al. Natural Course of Nonsevere Secondary Tricuspid Regurgitation. Journal of the American Society of Echocardiography. 2021; 34: 13-19.

[65] Utsunomiya H, Harada Y, Susawa H, Ueda Y, Izumi K, Itakura $\mathrm{K}$, et al. Tricuspid valve geometry and right heart remodelling: insights into the mechanism of atrial functional tricuspid regurgitation. European Heart Journal Cardiovascular Imaging. 2020; 21: 1068-1078.

[66] Margonato D, Ancona F, Ingallina G, Melillo F, Stella S, Biondi $\mathrm{F}$, et al. Tricuspid Regurgitation in Left Ventricular Systolic Dysfunction: Marker or Target? Frontiers in Cardiovascular Medicine. 2021; 8: 702589.

[67] Lee J, Song J, Park JP, Lee JW, Kang D, Song J. Long-term prognosis of isolated significant tricuspid regurgitation. Circulation Journal. 2010; 74: 375-380.

[68] Otto CM, Nishimura RA, Bonow RO, Carabello BA, Erwin JP 3rd, Gentile F, et al. 2020 ACC/AHA Guideline for the Management of Patients with Valvular Heart Disease: Executive Summary a Report of the American College of Cardiology/American Heart Association Joint Committee on Clinical Practice Guidelines. Circulation. 2021; 143: e35-e71.

[69] Hamandi M, Smith RL, Ryan WH, Grayburn PA, Vasudevan A, George TJ, et al. Outcomes of Isolated Tricuspid Valve Surgery have Improved in the Modern Era. The Annals of Thoracic Surgery. 2019; 108: 11-15.

[70] Vahanian A, Beyersdorf F, Praz F, Milojevic M, Baldus S, Bauersachs J, et al. $2021 \mathrm{ESC} / \mathrm{EACTS}$ Guidelines for the management of valvular heart diseaseDeveloped by the Task Force for the management of valvular heart disease of the European Society of Cardiology (ESC) and the European Association for Cardio-Thoracic Surgery (EACTS). European Heart Journal. 2021. (in press)

[71] Zoghbi WA, Adams D, Bonow RO, Enriquez-Sarano M, Foster E, Grayburn PA, et al. Recommendations for Noninvasive Evaluation of Native Valvular Regurgitation: a Report from the American Society of Echocardiography Developed in Collaboration with the Society for Cardiovascular Magnetic Resonance. Journal of the American Society of Echocardiography. 2017; 30: 303-371.

[72] Dahou A, Ong G, Hamid N, Avenatti E, Yao J, Hahn RT. Quantifying Tricuspid Regurgitation Severity: A Comparison of Proximal Isovelocity Surface Area and Novel Quantitative Doppler Methods. JACC: Cardiovascular Imaging. 2019; 12: 560-562.

[73] Öztürk C, Schueler R, Weber M, Nickenig G, Hammerstingl C. Comparison of different imaging modalities for the quantification of tricuspid valve geometry and regurgitation: a retrospective, single-center study. Health Science Reports. 2020; 3: 1-9.

[74] Hahn RT, Zamorano JL. The need for a new tricuspid regurgitation grading scheme. European Heart Journal Cardiovascular Imaging. 2017; 18: 1342-1343.

[75] Lang RM, Badano LP, Mor-Avi V, Afilalo J, Armstrong A, Er- nande L, et al. Recommendations for cardiac chamber quantification by echocardiography in adults: an update from the American Society of Echocardiography and the European Association of Cardiovascular Imaging. Journal of the American Society of Echocardiography. 2015; 28: 1-39.e14

[76] Lang RM, Badano LP, Tsang W, Adams DH, Agricola E, Buck $\mathrm{T}$, et al. EAE/ASE recommendations for image acquisition and display using three-dimensional echocardiography. Journal of the American Society of Echocardiography. 2012; 25: 3-46.

[77] Kovács A, Lakatos B, Tokodi M, Merkely B. Right ventricular mechanical pattern in health and disease: beyond longitudinal shortening. Heart Failure Reviews. 2019; 24: 511-520.

[78] Bernardo RJ, Haddad F, Couture EJ, Hansmann G, Perez VADJ, Denault AY, et al. Mechanics of right ventricular dysfunction in pulmonary arterial hypertension and heart failure with preserved ejection fraction. Cardiovascular Diagnosis and Therapy. 2020; 10: $1580-1603$.

[79] Fredriksson AG, Svalbring E, Eriksson J, Dyverfeldt P, Alehagen U, Engvall J, et al. 4D flow MRI can detect subtle right ventricular dysfunction in primary left ventricular disease. Journal of Magnetic Resonance Imaging. 2016; 43: 558-565.

[80] Hahn RT, Waxman AB, Denti P, Delhaas T. Anatomic Relationship of the Complex Tricuspid Valve, Right Ventricle, and Pulmonary Vasculature: A Review. JAMA Cardiology. 2019; 4: 478.

[81] Brener MI, Grayburn P, Lindenfeld J, Burkhoff D, Liu M, Zhou $\mathrm{Z}$, et al. Right Ventricular-Pulmonary Arterial Coupling in Patients with HF Secondary MR: Analysis From the COAPT Trial. JACC: Cardiovascular Interventions. 2021; 14: 2231-2242.

[82] Lurz P, Orban M, Besler C, Braun D, Schlotter F, Noack T, et al. Clinical characteristics, diagnosis, and risk stratification of pulmonary hypertension in severe tricuspid regurgitation and implications for transcatheter tricuspid valve repair. European Heart Journal. 2020; 41: 2785-2795.

[83] Badhwar V, Rankin JS, He M, Jacobs JP, Furnary AP, Fazzalari FL, et al. Performing Concomitant Tricuspid Valve Repair at the Time of Mitral Valve Operations is not Associated with Increased Operative Mortality. The Annals of Thoracic Surgery. 2017; 103: 587-593.

[84] David TE, David CM, Manlhiot C. Tricuspid annulus diameter does not predict the development of tricuspid regurgitation after mitral valve repair for mitral regurgitation due to degenerative diseases. The Journal of Thoracic and Cardiovascular Surgery. 2018; 155: 2429-2436.

[85] Dreyfus J, Audureau E, Bohbot Y, Coisne A, Lavie-Badie Y, Bouchery M, et al. TRI-SCORE: a new risk score for in-hospital mortality prediction after isolated tricuspid valve surgery. European Heart Journal. 2021. (in press)

[86] Kawsara A, Alqahtani F, Nkomo VT, Eleid MF, Pislaru SV, Rihal CS, et al. Determinants of Morbidity and Mortality Associated with Isolated Tricuspid Valve Surgery. Journal of the American Heart Association. 2021; 10: 1-18.

[87] Singh N, Kim H, Haydock DA. Isolated Tricuspid Valve Surgery: the Auckland Experience 2011-2019. Heart Lung and Circulation. 2021. (in press)

[88] Dreyfus J, Flagiello M, Bazire B, Eggenspieler F, Viau F, Riant $\mathrm{E}$, et al. Isolated tricuspid valve surgery: impact of aetiology and clinical presentation on outcomes. European Heart Journal. 2020; 41: 4304-4317. 